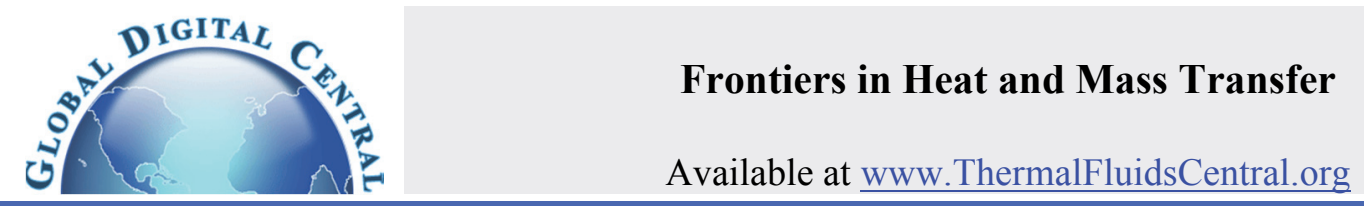

\title{
TWO DIFFERENT APPROACHES FOR ANALYSING HEAT TRANSFER IN A POWER-LAW FLUID FLOW BETWEEN PARALLEL PLATES
}

\author{
Fábio A. Caldas ${ }^{\mathrm{a}^{*}}$, Paulo M. Coelho ${ }^{\mathrm{b}, \uparrow}$ \\ ${ }^{a}$ Departamento de Engenharia Mecânica, Faculdade de Engenharia da Universidade do Porto, Porto, 4200-465, Portugal \\ ${ }^{b}$ CEFT, Departamento de Engenharia Mecânica, Faculdade de Engenharia da Universidade do Porto, Porto, 4200-465, Portugal
}

\begin{abstract}
In this paper, Nusselt numbers for a power-law fluid in a fully developed laminar flow between parallel plates with constant, and different, wall heat fluxes in the presence of dissipation effects are presented. The Nusselt numbers values were obtained following two different approaches. One is the "classical" approach, based on a single bulk temperature, and this approach is used in this work to obtain for the first time generic analytical expressions for Nusselt numbers. In the new approach, different bulk temperatures are used for each $\mathrm{Nu}^{\prime}$ determination, one bulk temperature for each side of the location of the temperature profile where the derivative $\partial T / \partial y=0$.

Keywords: Convection, viscous dissipation, analytical solution, two bulk temperatures.
\end{abstract}

\section{INTRODUCTION}

An analytic study regarding heat transfer in a fully developed laminar flow between parallel plates of a power-law fluid with constant, but different, wall heat fluxes, in the presence of viscous dissipation, is presented in this work. As it is explained in detail below, the same subject has been partially addressed in the literature previously but not in the precise manner undertaken here which, as we will show, may be advantageous.

In the review article of Hartnett and Kostic (1989), restricted to the hydrodynamics and heat transfer aspects of non-Newtonian flow in rectangular duct geometries, the analytical solution to this problem presented by the authors was due to Skelland (1967), which is only valid for constant and equal heat fluxes at the walls in the absence of viscous dissipation. In the review work of Lawal and Mujumdar (1987) where an overview of laminar duct flow and heat transfer regarding purely viscous non-Newtonian fluids taking into account viscous dissipation and the effect of variables properties is presented, again just the particular case of constant and symmetric wall heat fluxes is discussed. Etemad and Majumdar (1994) carried out a numerical study regarding the simultaneously developing laminar flow and heat transfer of a power-law fluid flowing between two parallel plates. Several different thermal boundary conditions were examined. They showed that the Nusselt number is significantly affected by the variation of the fluid viscosity with temperature, viscous dissipation, the power-law index value as well as the fluid Prandtl number and thermal boundary conditions.

Only recently was the asymmetric wall heat fluxes case addressed analytically in the literature by Tso et al. (2010). In this work the authors present analytical solutions for the temperature profiles and Nusselt numbers, $\mathrm{Nu}$, but, because they use only one coordinate system in the mathematical development, the general result is, as admitted to by the authors themselves, too complex. Simpler expressions for four specific values of the power-law index, $n$, namely $0.25,0.5,1$ and 2 , are then presented by the authors in order to revel the heat transfer characteristics, but the only verification done was via results from the literature for $n=1$. For the particular case of equal heat fluxes at the walls in the absence of viscous dissipation, the $\mathrm{Nu}$ values given by those simpler expressions, based on the hydraulic diameter, for $n=0.25$, $n=0.5$ and $n=2$, are 8.0, 6.9 and 6.3 , respectively. Those values are different from the corresponding ones in the literature, Skelland (1967) and Baptista et al. (2013), that for the same power-law index values are of 9.5, 8.8 and 7.9, respectively, which may indicate a problem in this analytical solution. Also, by not using the generalized Brinkman number definition, $\mathrm{Br}^{*}$, Coelho and Pinho (2009), the Nusselt number values rapidly decrease with an increase of the Brinkman number, something that, as shown in Coelho and Faria (2011), hinders the graphical representations of $\mathrm{Nu}$.

Considering all of the studies discussed above, it is fair to state there is currently no simple and generic analytical solution for the Nusselt numbers, $\mathrm{Nu}$, i.e., valid for any values of the power index, $n$, wall heat flux ratio, $\boldsymbol{\Phi}=\dot{q}_{\mathrm{w}, 1} / \dot{q}_{\mathrm{w}, 2}$, and generalized Brinkman number. Such an analytical solution and the underlying mathematical approach, is one of the main contributions of the present work.

The current study starts by showing the calculation procedure that leads to the analytical expressions of the Nusselt numbers at the duct walls. Figure 1 shows schematically the plane walls 1 and 2 of the duct, spaced apart by a distance of $2 H$, with the wall heat fluxes applied, $\dot{q}_{\mathrm{w}, 1}$ and $\dot{q}_{\mathrm{w}, 2}$, respectively. The coordinate systems used and an asymmetric temperature profile are also shown. By using two coordinate systems, $y_{\mathrm{I}}$ and $y_{\mathrm{II}}$, cf. Fig. 1, the $y$ variable in the velocity profile remains always positive, allowing an easy integration of the differential equations for any value of $n$ and giving rise to simpler mathematical expressions. As far as the authors are aware, the use of two coordinate systems in this type of problems is also new to the literature and here we demonstrate its utility to such problems. The wall temperatures, $T_{\mathrm{w}, 1}$ and $T_{\mathrm{w}, 2}$, represented in Fig. 1, are naturally a function of the longitudinal coordinate $x$.

\footnotetext{
*Trainee at EDP, Lisbon, 1200-109, Portugal. Email: fabio.caldas@edp.pt

${ }^{\dagger}$ Corresponding author.Email: pmc@fe.up.pt
} 


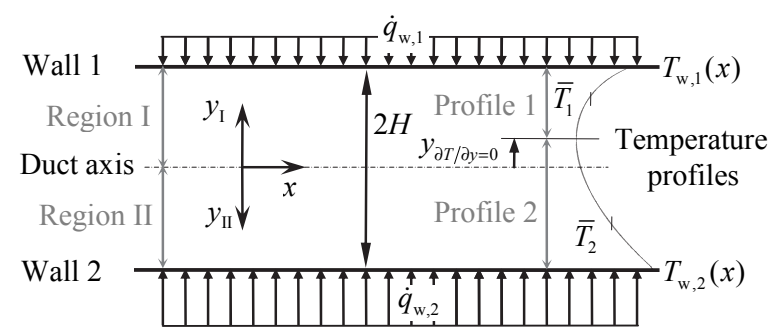

Fig. 1 Schematic representation of the parallel plates duct, the boundary conditions and of the coordinate systems. A temperature profile is also shown, which is divided by the point where $\partial \mathrm{T} / \partial \mathrm{y}=0$.

For the analytical expressions of the Nusselt numbers at the walls 1 and 2, $\mathrm{Nu}_{1}$ and $\mathrm{Nu}_{2}$ respectively, the "classical" approach was used, i.e., a single bulk temperature was considered for the entire duct crosssection. When the temperature profile is asymmetric, this approach may lead to negative Nusselt number values and discontinuities in the $\mathrm{Nu}$ curves and, as will be seen in section 3 , even to a case where $\mathrm{Nu}=4$ regardless the values of $n$ or the heat flux ratio, $\Phi=\dot{q}_{\mathrm{w}, 1} / \dot{q}_{\mathrm{w}, 2}$.

In order to obtain Nusselt number values free of the above mention anomalies, what we will term " $\mathrm{Nu}^{\prime}$ ", and therefore comparable with the existing values in the literature for cases where a symmetric temperature profile exists, e.g. pipe flow, it is necessary to use two different bulk temperatures, $\bar{T}_{1}$ and $\bar{T}_{2}$, cf. Fig. 1 , to calculate the two Nusselt numbers, $\mathrm{Nu}_{1}^{\prime}$ and $\mathrm{Nu}_{2}^{\prime}$ respectively. In section 3.1 , the results of the Nusselt numbers obtained with this new approach, where a bulk temperature is calculated for each side of the location of the temperature profile where $\partial T / \partial y=0, \quad y=y_{\partial T / \partial y=0}$ are shown and discussed. This location, $y=y_{\partial T / \partial y=0}$, effectively divides the duct cross-section in two independent zones, each one with a temperature profile, named Profile 1 and Profile 2 in Fig. 1, since there is no heat transfer between the two zones. The use of this new approach in the flow between parallel plates is an additional contribution of the present work.

In a flow with a symmetrical temperature profile, the proposed approach reproduces $\mathrm{Nu}^{\prime}$ values equal to the ones obtained using the "classical" approach. Therefore, it can be stated that in situations with asymmetric temperature profiles, the Nusselt numbers obtained using the new approach are also comparable with the $\mathrm{Nu}$ values of the literature cases where the temperature profile is symmetric.

Generally, this new approach can be used whenever the temperature profile is asymmetric, e.g. in annular flow, where the flow between parallel plates is a limiting case, as explained in the work of Coelho and Poole (2017).

\section{CALCULATION PROCEDURE}

In a fully developed laminar flow between parallel plates of a powerlaw fluid, the dimensionless velocity profile is given by the following equation,

$u^{*}=\frac{u}{\bar{U}}=\frac{2 n+1}{n+1}\left(1-y^{* \frac{n+1}{n}}\right)$

where $u$ is the local velocity, $\bar{U}$ is the bulk velocity and $y^{*}=y / H$ is the transversal coordinate in dimensionless form, Fig. 1.

The differential form of the energy conservation equation in Cartesian coordinates, for a fully developed flow between parallel plates in the presence of viscous dissipation, is shown in Eq. (2), $k \frac{\partial^{2} T}{\partial y^{2}}-\tau_{y x} \frac{d u}{d y}=\rho c u \frac{\partial T}{\partial x}$

where $T$ is the temperature, $x$ the longitudinal coordinate, Fig. 1, $\rho, c$ and $k$ are density, specific heat and conductivity of the fluid, respectively, and $\tau_{y x}$ is the absolute value of the local shear stress.

Using a similar method to the one shown in Çengel and Turner (2005) for a constant wall heat flux in a pipe, but considering the presence of viscous dissipation, it can also be shown that,

$\frac{\partial T}{\partial x}=\frac{d T_{\mathrm{w}}}{d x}=\frac{d \bar{T}}{d x}=\frac{\overline{\dot{q}}_{\mathrm{w}}+\bar{U} \tau_{\mathrm{w}}}{\rho \bar{U} H c}=$ constant

where $\bar{T}$ is the bulk temperature, $\overline{\dot{q}}_{\mathrm{w}}$ is the average wall heat flux, $\overline{\dot{q}}_{\mathrm{w}}=\left(\dot{q}_{\mathrm{w}, 1}+\dot{q}_{\mathrm{w}, 2}\right) / 2$, and $\tau_{\mathrm{w}}$ is the wall shear stress.

Replacing $\partial T / \partial x$ by $d \bar{T} / d x$ in equation (2) leads to the following dimensionless equation,

$\frac{d^{2} T^{*}}{d y^{* 2}}-2 \tau^{*} \mathrm{Br}^{*} \frac{d u^{*}}{d y^{*}}=\frac{\mathrm{Pe}}{16} u^{*} \frac{d \bar{T}^{*}}{d x^{*}}$

where $T^{*}=\left(T-T_{0}\right) k /\left(D_{\mathrm{h}} \overline{\dot{q}}_{\mathrm{w}}\right)$ is the dimensionless temperature $\left(T_{0}\right.$ is a reference bulk temperature and $D_{\mathrm{h}}=4 H$ the hydraulic diameter), $\tau^{*}$ is the dimensionless local shear stress $\tau^{*}=\tau_{y x} / \tau_{\mathrm{w}}=y / H=y^{*}, \mathrm{Br}^{*}$ is the generalized Brinkman number, $\mathrm{Br}^{*}=\bar{U} \tau_{\mathrm{w}} /\left(8 \overline{\dot{q}}_{\mathrm{w}}\right)$ (Coelho and Pinho, 2009), $\mathrm{Pe}$ is the Péclet number, $\mathrm{Pe}=\mathrm{Re} \cdot \mathrm{Pr}=\rho \bar{U} D_{\mathrm{h}} c / k$ and $x^{*}$ is the dimensionless longitudinal coordinate, $x^{*}=x^{4} / P_{d x^{*}}=\left(1+8 \mathrm{Br}^{*}\right) 4 / \mathrm{Pe}$

The dimensionless form of Eq. (3), $\tau^{*}=y^{*}$, yields the following replaced in Eq. (4), and knowing that $\tau=y$, yields the following
expression for the differential form of the energy conservation equation,

$\frac{d^{2} T^{*}}{d y^{* 2}}-2 y^{*} \mathrm{Br}^{*} \frac{d u^{*}}{d y^{*}}=\frac{u^{*}}{4}\left(1+8 \mathrm{Br}^{*}\right)$

By replacing the expressions of $u^{*}$ and $d u^{*} / d y^{*}$ in Eq. (5), the final form of the energy equation is obtained, Eq. (6), where $\alpha=2 \mathrm{Br}^{*}(2 n+1) / n, \quad \beta=\left(1+8 \mathrm{Br}^{*}\right)(2 n+1) /(4 n+4) \quad$ and $\mathcal{E}=(n+1) / n$ are used in order to simplify the expressions.

$\frac{\partial^{2} T^{*}}{\partial y^{* 2}}=\beta\left(1-y^{* \varepsilon}\right)-\alpha y^{* \varepsilon}$

Since $y^{*}$ is raised to the power $(n+1) / n$, it must be always positive in order to make the integration of Eq. (6) possible. To assure this, two coordinate systems are used. The $x$-axis is the same while the $y$-axis are distinct, $y_{\mathrm{I}}$ and $y_{\mathrm{II}}$ axis, one for each region I and II, separated by the duct symmetry plane as shown in Fig. 1. Equation (6) is then integrated in both regions I and II, subject to the following boundary conditions,

$\left.k \frac{\partial T}{\partial y_{\mathrm{I}}}\right|_{y_{1}=H}=\left.\dot{q}_{\mathrm{w}, 1} \Leftrightarrow \frac{d T^{*}}{d y_{\mathrm{I}}^{*}}\right|_{y_{1}^{*}=1}=\frac{\boldsymbol{\Phi}}{2(\boldsymbol{\Phi}+1)}=\boldsymbol{\varphi} \cdot \boldsymbol{\Phi}$

$\left.T\right|_{y_{1}=H}=\left.T_{\mathrm{w}, 1} \Leftrightarrow T^{*}\right|_{y_{1}^{*}=1}=T_{\mathrm{w}, 1}^{*}$ 
$\left.\frac{\partial T}{\partial y_{\mathrm{I}}}\right|_{y_{1}=0}=-\left.\left.\frac{\partial T}{\partial y_{\mathrm{II}}}\right|_{y_{\mathrm{II}}=0} \Leftrightarrow \frac{d T^{*}}{d y_{\mathrm{I}}^{*}}\right|_{y_{\mathrm{I}}^{*}=0}=-\left.\frac{d T^{*}}{d y_{\mathrm{II}}^{*}}\right|_{y_{\mathrm{II}}^{*}=0}$

$\left.T\right|_{y_{1}=0}=\left.\left.T\right|_{y_{\mathrm{I}}=0} \Leftrightarrow T^{*}\right|_{y_{\mathrm{I}}^{*}=0}=\left.T^{*}\right|_{y_{\|}^{*}=0}$

$\left.k \frac{\partial T}{\partial y_{\mathrm{II}}}\right|_{y_{11}=H}=\left.\dot{q}_{\mathrm{w}, 2} \Leftrightarrow \frac{d T^{*}}{d y_{\mathrm{II}}^{*}}\right|_{y_{\mathrm{II}}^{*}=1}=\frac{1}{2(\Phi+1)}=\varphi$

The boundary conditions given by Eqs. (7) and (8), on one hand, and the boundary conditions given by Eqs. (9) and (10) on the other, allow the temperature profile equations in the regions I and II, respectively, to be obtained. The boundary condition (11) was used to validate the resulting expressions for $d T^{*} / d y^{*}$.

\section{RESULTS}

The integration of Eq. (6) in the regions I and II, cf. Fig. 1, leads to the following expressions for the temperature profiles,

$$
\begin{aligned}
& T_{\mathrm{I}}^{*}=-\frac{\eta y_{\mathrm{I}}^{* \varepsilon+2}}{\varepsilon+2}+\frac{\beta y_{\mathrm{I}}^{* 2}}{2}+y_{\mathrm{I}}^{*}(\varphi \Phi+\sigma)-\varphi \Phi-\frac{\beta}{2}+\frac{\eta}{\varepsilon+2}-\sigma+T_{\mathrm{w}, 1}^{*} \\
& T_{\mathrm{II}}^{*}=-\frac{\eta y_{\mathrm{II}}^{* \varepsilon+2}}{\varepsilon+2}+\frac{\beta y_{\mathrm{II}}^{* 2}}{2}-y_{\mathrm{II}}^{*}(\varphi \Phi+\sigma)-\varphi \Phi-\frac{\beta}{2}+\frac{\eta}{\varepsilon+2}-\sigma+T_{\mathrm{w}, 1}^{*}
\end{aligned}
$$

with $\sigma=(\alpha-\beta \varepsilon) /(\varepsilon+1)$ and $\eta=(\alpha+\beta) /(\varepsilon+1)$. By defining $y_{\mathrm{II}}^{*}=1$ in Eq. (13), the following expression for the wall 2 temperature, $T_{\mathrm{w}, 2}^{*}$, is obtained,

$$
T_{\mathrm{w}, 2}^{*}=-\frac{\Phi}{\Phi+1}+\frac{1}{2}+T_{\mathrm{w}, 1}^{*}
$$

Although $T_{\mathrm{w}, 1}^{*}$ is unknown and a function of $x^{*}$, which requires the use of a temperature difference in a graphical representation of the temperature profiles, for example $T^{*}-T_{\mathrm{w}, 1}^{*}$, this does not affect the Nusselt number calculations, since these are based on a temperature difference that is always independent of the wall temperatures, as will be demosntrated below.

The derivative of the temperature profile in the duct axis is given by the following expression,

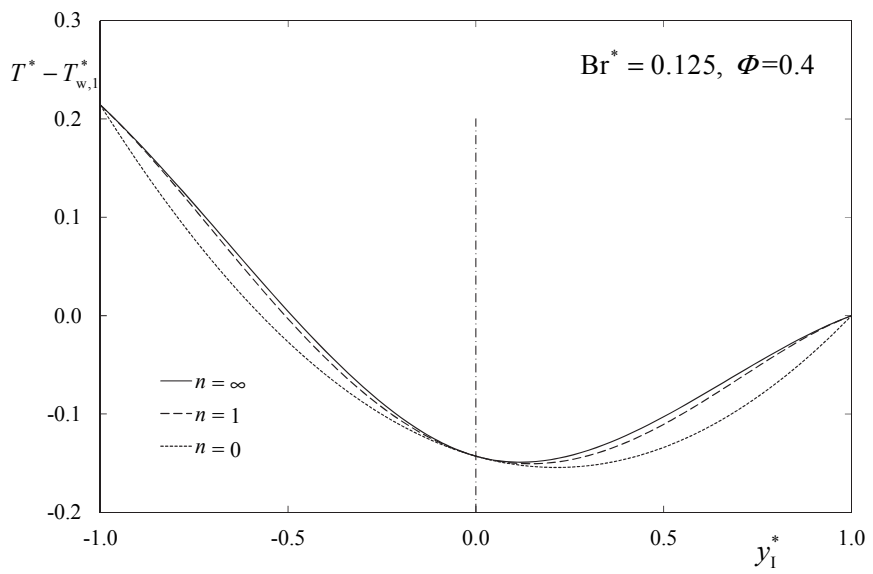

Fig. 2 Dimensionless temperature profiles for three different values of $n, \mathrm{Br}^{*}=0.125$ and $\Phi=0.4$. The vertical line represents the duct axis. $\left.\frac{\partial T^{*}}{\partial y_{1}^{*}}\right|_{y_{1}^{*}=0}=\frac{1}{4}-\frac{1}{2(\Phi+1))}$

as it can be observed, the expression is independent of $n$ and $\mathrm{Br}^{*}$, since these two variables have a symmetrical effect on the temperature profile. The temperature at the duct axis is generally a function of the variables $n, \mathrm{Br}^{*}$ and $\Phi$, except for the case where the heat supplied at the duct walls equals the heat generated by viscous dissipation, i.e., $\mathrm{Br}^{*}=0.125$. In this case this temperature is given by the following expression,

$\left.T^{*}\right|_{y=0}=\frac{1}{2(\Phi+1)}-\frac{1}{2}+T_{\mathrm{w}, 1}^{*}$

being in this particular case also independent of $n$.

Figure 2 shows three temperature profiles for three representative power-law index values, $(0,1$ and $\infty), \mathrm{Br}^{*}=0.125$ and $\Phi=0.4$ which illustrate these points.

To calculate the Nusselt numbers, $\mathrm{Nu}_{1}=h_{1} D_{\mathrm{h}} / k$ and $\mathrm{Nu}_{2}=h_{2} D_{\mathrm{h}} / k$, where $h_{i}$ is the convection coefficient, the expressions (17) and (18) were used, respectively. They arise via rendering dimensionless the equation $\dot{q}_{\mathrm{w}, i}=h_{i}\left(T_{\mathrm{w}, i}-\bar{T}\right)$ for each wall.

$\dot{q}_{\mathrm{w}, 1}=h_{1}\left(T_{\mathrm{w}, 1}-\bar{T}\right) \Leftrightarrow \mathrm{Nu}_{1}=\frac{h_{1} D_{\mathrm{h}}}{k}=\frac{2 \Phi}{1+\Phi} \frac{1}{\left(T_{\mathrm{w}, 1}^{*}-\bar{T}^{*}\right)}$

$\dot{q}_{\mathrm{w}, 2}=h_{2}\left(T_{\mathrm{w}, 2}-\bar{T}\right) \Leftrightarrow \mathrm{Nu}_{2}=\frac{h_{2} D_{\mathrm{h}}}{k}=\frac{2}{1+\Phi} \frac{1}{\left(T_{\mathrm{w}, 2}^{*}-\bar{T}^{*}\right)}$

The bulk temperature, $\bar{T}$, used in the Nusselt numbers calculation was obtained through the following integral,

$\bar{T}=\frac{\int_{-H}^{H} u b T d y}{\bar{U} 2 H b} \Leftrightarrow \bar{T}^{*}=\frac{1}{2} \int_{0}^{1} u^{*} T^{*} d y_{\mathrm{I}}^{*}+\frac{1}{2} \int_{0}^{1} u^{*} T^{*} d y_{\mathrm{II}}^{*}$

where $b$ is the duct spanwise length. The analytical expression for this bulk temperature is given by Eq. (20).

$\bar{T}^{*}=T_{\mathrm{w}, 1}^{*}-\varphi \Phi-\frac{\beta(\varepsilon+4)}{3(\varepsilon+3)}-\frac{2 \varepsilon^{2} \sigma+\varepsilon(9 \sigma-2 \eta)-4 \eta+9 \sigma}{(\varepsilon+3)(2 \varepsilon+3)}$

The mathematical expressions for the Nusselt numbers at both walls, $\mathrm{Nu}_{1}$ and $\mathrm{Nu}_{2}$, are given by Eqs. (21) and (22), respectively.

$$
\begin{aligned}
& \mathrm{Nu}_{1}= \\
& \frac{12 \Phi\left(20 n^{2}+13 n+2\right)}{4 \mathrm{Br}^{*}(\Phi+1)\left(14 n^{2}+11 n+2\right)+2 \Phi\left(23 n^{2}+14 n+2\right)-\left(14 n^{2}+11 n+2\right)} \\
& \mathrm{Nu}_{2}= \\
& \frac{12\left(20 n^{2}+13 n+2\right)}{4 \mathrm{Br}^{*}(\Phi+1)\left(14 n^{2}+11 n+2\right)-\Phi\left(14 n^{2}+11 n+2\right)+2\left(23 n^{2}+14 n+2\right)}
\end{aligned}
$$

The validation of Eqs. (21) and (22), was carried out as it follows: by making $\Phi=1$ both equations reduce to the same expression of the Nusselt number, that is equal to the expression presented in Baptista et al. (2013), their Eq. (38); imposing $\Phi=1$ and $\mathrm{Br}^{*}=0$, on the Nusselt number equations, the obtained expression is equal to the $\mathrm{Nu}$ equation presented in Skelland (1967); by making $\mathrm{Br}^{*}=0$ and $n=1$ in Eqs. (21) and (22) the following expressions are obtained, 
$\mathrm{Nu}_{1}=140 \Phi /(26 \Phi-9)$ and $\mathrm{Nu}_{2}=140 /(26-9 \Phi)$, respectively, in accordance with the expressions provided in Shah and London (1978); finally, if in one of the previous equations, Eq. (21) or Eq. (22), the variable $\Phi$ is replaced by $1 / \Phi$, the other equation, Eq. (22) or Eq. (21) respectively, is obtained, something that is expected in a hydrodynamically symmetrical flow. It is also because of this particularity that in the present work the variable $\Phi$ varies between zero and one, since both expressions of $\mathrm{Nu}_{1}$ and $\mathrm{Nu}_{2}$, together, show the effect of $\Phi$ in the range between zero and infinity

Figures 3, 4 and 5 show plots of Nusselt numbers, $\mathrm{Nu}_{1}$ and $\mathrm{Nu}_{2}$, versus the independent variables $n, \Phi$ and $\mathrm{Br}^{*}$, respectively. Fig. 3 shows the typical behaviour of $\mathrm{Nu}_{1}$ and $\mathrm{Nu}_{2}$ with the power law index, $n$, for different Brinkman numbers when $\Phi$ is kept constant, in this case $\Phi=0.4$. For low values of $\mathrm{Br}^{*}$, when $n$ decreases, the velocity profile approaches a plug profile, with higher velocities near the walls, while the Nusselt numbers increase. For higher values of $\mathrm{Br}^{*}$ however, the viscous dissipation effect, which occurs closer to the walls with the decrease of $n$, surpasses the effect of the increased velocity near the walls and $\mathrm{Nu}$ decreases. In the case of $\mathrm{Nu}_{1}$ and $\mathrm{Br}^{*}=0$, Fig. 3a), with the decrease of the value of $n$, the wall temperature, $T_{\mathrm{w}, 1}^{*}$, becomes higher than the "classical" bulk temperature, $\bar{T}^{*}$, causing a discontinuity in the $\mathrm{Nu}_{1}$ curve and negative values of this Nusselt number.

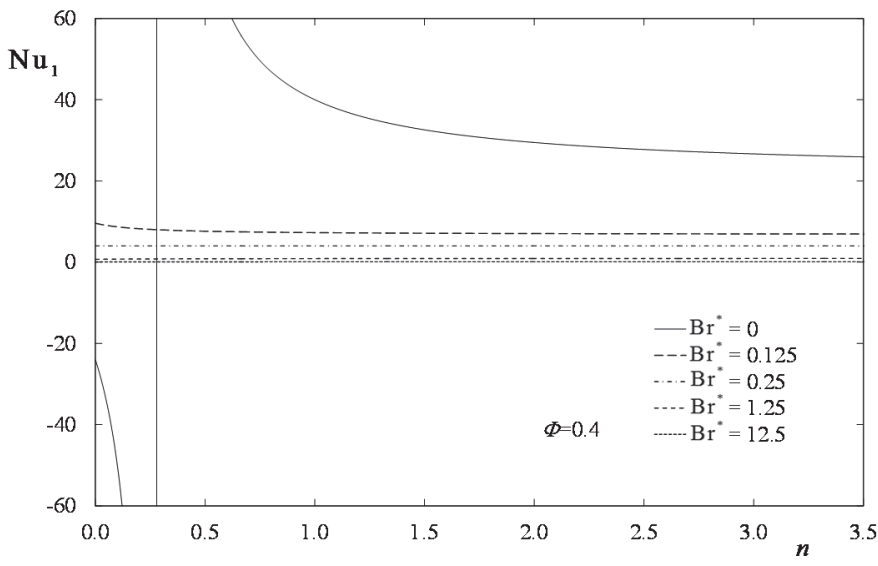

(a) $\mathrm{Nu}_{1}$

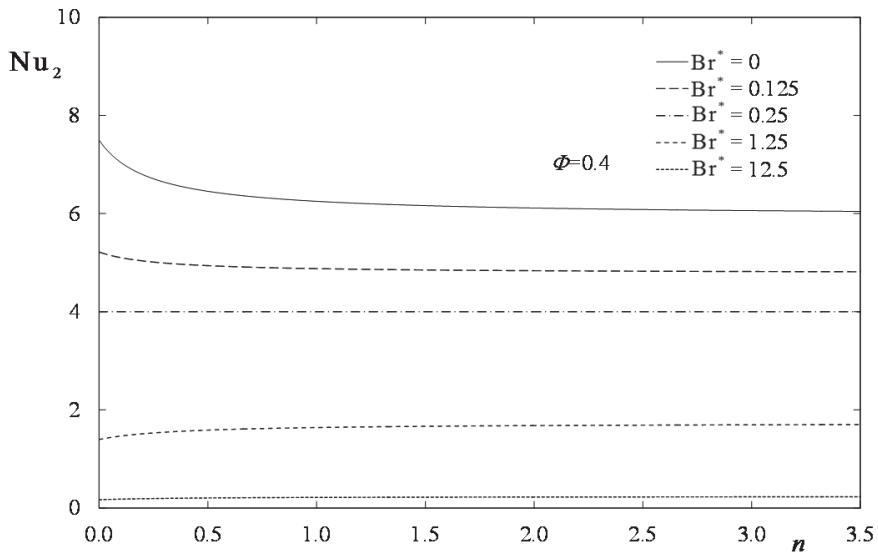

(b) $\mathrm{Nu}_{2}$

Fig. 3 Nusselt numbers versus the power-law index, $n$, for $\Phi=0.4$ and different values of the Brinkman number, $\mathrm{Br}^{*}$. Data obtained using the "classical" approach for the bulk temperature calculation.

Figure 4 shows the variation of $\mathrm{Nu}_{1}$ and $\mathrm{Nu}_{2}$ with $\Phi$ for different values of $\mathrm{Br}^{*}$ and $n=0.5$. For wall heat flux ratios, $\Phi$, in the range
$0<\Phi<1, \mathrm{Nu}_{2}$ is always positive, since $T_{\mathrm{w}, 2}^{*}>\bar{T}^{*}$, see Fig. $4 \mathrm{~b}$ ). For both walls, it is clear that an increase of $\mathrm{Br}^{*}$ results in a reduction of the Nusselt numbers.

When $\Phi$ decreases, meaning that $\dot{q}_{\mathrm{w}, 1}$ decreases in relation to $\dot{q}_{\mathrm{w}, 2}$, the wall temperature $T_{\mathrm{w}, 1}^{*}$ also decreases, becoming equal to, and then lower than, the bulk temperature, $\bar{T}^{*}$, this leads to infinite and negative values of $\mathrm{Nu}_{1}$, Eq. (17), cf. Fig. 4a). For higher values of the Brinkman number, this discontinuity in $\mathrm{Nu}_{1}$ occurs at lower values of $\Phi$. The value of $\Phi$ for which $T_{\mathrm{w}, 1}^{*}=\bar{T}^{*}, \Phi_{T_{\mathrm{w}, 1}^{*}=\bar{T}^{*}}$, is given by Equation (23).

$\Phi_{T_{\mathrm{w}, 1}^{*}=\bar{T}^{*}}=\frac{1-4 \mathrm{Br}^{*}}{\left[4 \mathrm{Br}^{*}+\frac{46 n^{2}+28 n+4}{14 n^{2}+11 n+2}\right]}$

Equation (23) shows that regardless of the value of $n$, for $\mathrm{Br}^{*}>0.25$ the wall temperature $T_{\mathrm{w}, 1}^{*}$ is always higher than the bulk temperature,

$\bar{T}^{*}$, regardless of the positive value of $\Phi$.

The variation of $\mathrm{Nu}_{1}$ and $\mathrm{Nu}_{2}$ with $\mathrm{Br}^{*}$ for different values of $\Phi$ and $n=0.5$ is shown in Fig. 5. In general, it can be seen, once again, that an increase in the Brinkman number values leads to a reduction in the Nusselt numbers and for wall heat flux ratios between $0<\Phi<0.5$, according to Eq. (23), the $\mathrm{Nu}_{1}$ values may become infinity and negative.

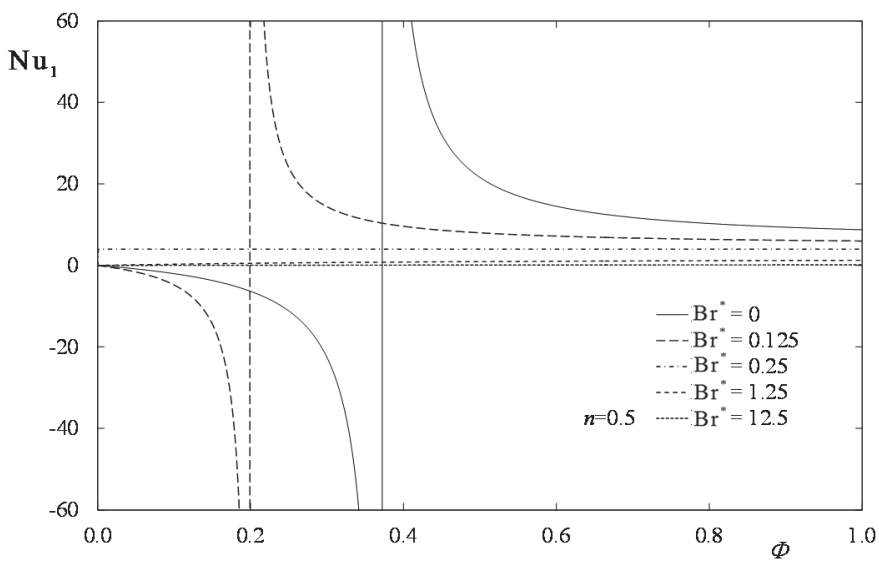

(a) $\mathrm{Nu}_{1}$

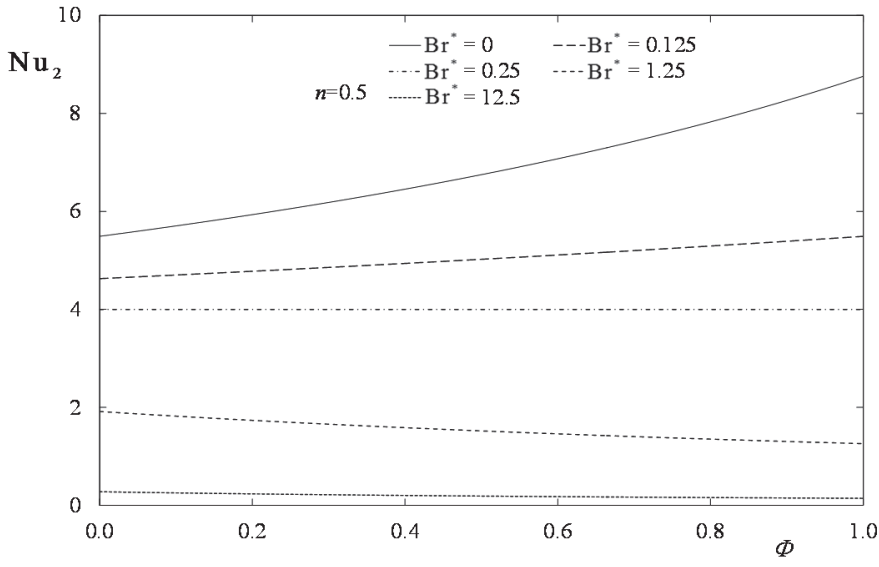

(b) $\mathrm{Nu}_{2}$

Fig. 4 Nusselt numbers versus the wall heat flux ratio, $\Phi$, for $n=0.5$ and different values of the Brinkman number, $\mathrm{Br}^{*}$. Data obtained using the "classical" approach for the bulk temperature calculation. 
An interesting fact noted when analyzing Fig. 3, 4, and, 5, is that for $\mathrm{Br}^{*}=0.25$, i.e., $\dot{q}_{\text {visc dissip }} / \overline{\dot{q}}_{\mathrm{w}}=2$, both Nusselt numbers are equal, $\mathrm{Nu}_{1}=\mathrm{Nu}_{2}$ regardless of the values of $n$ or $\Phi$. In fact, when replacing $\mathrm{Br}^{*}$ by 0.25 in Eqs. (21) or (22) the result is $\mathrm{Nu}_{1}=\mathrm{Nu}_{2}=4$. The use of a single bulk temperature for the entire duct cross-section is responsible for this outcome and again shows that the heat transfer coefficients thus obtained are far from the real value. Essentially, if correct, this would mean the "real" convection coefficient is simultaneously independent of velocity and temperature profiles as varied when $n$ and $\Phi$ vary between zero to infinity, i.e., a velocity profile varying between plug flow and almost triangular.

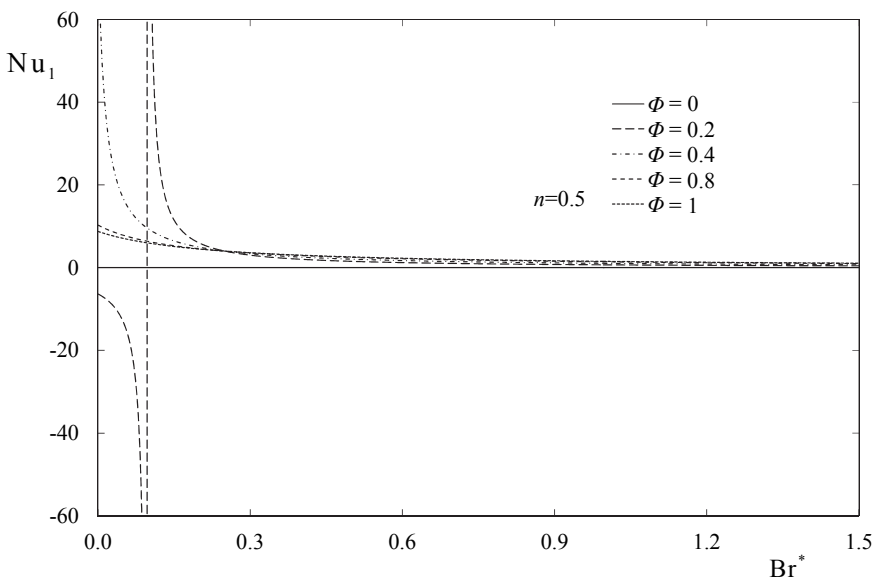

(a) $\mathrm{Nu}_{1}$

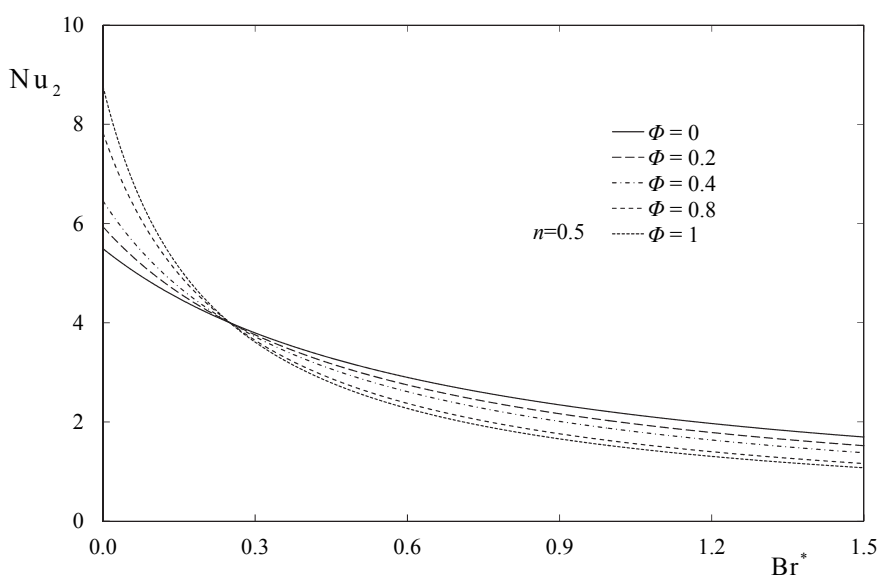

(b) $\mathrm{Nu}_{2}$

Fig. 5 Nusselt numbers versus the Brinkman number, $\mathrm{Br}^{*}$, for $n=0.5$ and different values of the wall heat flux ratio, $\Phi$. Data obtained using the "classical" approach for the bulk temperature calculation.

The results presented in Figs 3-5 show that the "classical" approach, i.e., the use of a single bulk temperature for the whole duct crosssection, while useful from a practical standpoint, since this temperature is easily determined, experimentally and mathematically, sometimes yields Nusselt numbers that deviate from the values and behaviours of the expected heat transfer coefficient. By correctly relating the wall temperature with the bulk temperature, which, obviously, is the most relevant in practice, the "classical" approach is an important tool and it will continue to be used.

The next section presents a different approach for the Nusselt numbers calculation that eliminates the singularities referred to above, while allowing a direct comparison between the $\mathrm{Nu}^{\prime}$ values thus obtained, regardless of the temperature profile shape, with those in the literature for the cases where the temperature profile is symmetrical.

\section{1 $\mathrm{Nu}_{1}^{\prime}$ and $\mathrm{Nu}_{2}^{\prime}$ Calculated Using Two Bulk Temperatures}

In this new approach for the Nusselt number calculation, two bulk temperatures are considered, one for each side of the location of the temperature profile where $\partial T / \partial y=0, y=y_{\partial T / \partial y=0}$, cf. Fig. 1 . In the case of a symmetrical temperature profile, both approaches produce the same values for the Nusselt numbers.

Equations (24) and (25) are the expressions of the bulk temperatures for the two temperature profiles, cf. Fig. $1, \bar{T}_{\mathrm{w}, 1}^{*}$ e $\bar{T}_{\mathrm{w}, 2}^{*}$, respectively, since in the present case $0<\Phi<1$ the coordinate $y_{d T^{*} / d y^{*}=0}^{*}$ is always in region $\mathrm{I}$.

$$
\begin{aligned}
& \bar{T}_{1}^{*}=\frac{\int_{y_{1, d T^{*} / d y^{*}=0}^{*}}^{1} u^{*} T^{*} d y_{\mathrm{I}}^{*}}{\int_{y_{1, d T^{*} / d y^{*}=0}^{*}}^{1} u^{*} d y_{\mathrm{I}}^{*}} \\
& \bar{T}_{2}^{*}=\frac{\int_{0}^{1} u^{*} T^{*} d y_{\mathrm{II}}^{*}+\int_{0}^{y_{\mathrm{L}}^{*} d T^{*} / d y^{*}=0} u^{*} T^{*} d y_{\mathrm{I}}^{*}}{\int_{0}^{1} u^{*} d y_{\mathrm{II}}^{*}+\int_{0}^{y_{\mathrm{I}}^{*} d T^{*} / d y^{*}=0} u^{*} d y_{\mathrm{I}}^{*}}
\end{aligned}
$$

The new Nusselt numbers are calculated using Eqs. (17) and (18), with $\bar{T}^{*}$ being replaced by $\bar{T}_{1}^{*}$ and $\bar{T}_{2}^{*}$, respectively.

The coordinate $y_{\mathrm{I}, d T^{*} / d y^{*}=0}^{*}$ was calculated as the root of Eq. (26) that only has analytical solutions for $n=0, n=0.5$ and $n=1$, while for other $n$ values a numerical solution was used.

$$
\frac{d T^{*}}{d y_{\mathrm{I}}^{*}}=-\frac{y_{\mathrm{I}}^{*(\varepsilon+1)}(\alpha+\beta)}{\varepsilon+1}+\beta y_{\mathrm{I}}^{*}+\varphi \Phi+\frac{\alpha-\beta \varepsilon}{\varepsilon+1}
$$

The relationship between both convection coefficients, given by the "classical" and the new approach, is shown in Equation (27), also valid for the Nusselt numbers. This equation was deduced knowing that the wall heat flux, $\dot{q}_{\mathrm{w}, i}$, is the same regardless of the approach used.

$$
\begin{aligned}
& \dot{q}_{\mathrm{w}, i}= \\
& h_{\text {(unconventional approach) }}^{\prime}\left(T_{\mathrm{w}, i}-\bar{T}_{i}\right)=h_{\text {(classical approach) }}\left(T_{\mathrm{w}, i}-\bar{T}\right)
\end{aligned}
$$

Equation (27) shows that both approaches allow correct calculations of the wall temperature, $T_{\mathrm{w}, i}$, given the corresponding Nusselt number and bulk temperature, $\bar{T}_{i}$ or $\bar{T}$. The advantage of the new approach, as previously mentioned, is that it allows more realistic heat transfer coefficients to be obtained, comparable with the existing values in the literature for flows inside ducts when the temperature profile is symmetrical. Although the new bulk temperatures have these advantages, their calculation is not straightforward potentially hindering its use.

Figures 6,7 and 10, show the results already presented in figures 3 to 5 but now obtained through the use of the new approach, i.e., using different bulk temperatures for calculating the Nusselt number on each wall.

Figure 6 shows the variation of the Nusselt numbers, $\mathrm{Nu}_{1}^{\prime}$ and $\mathrm{Nu}_{2}^{\prime}$, with the power law index $n$, for different values of Brinkman number and $\Phi=0.4$. Nusselt numbers show a variation similar to the one observed in Fig. 3, but now the $\mathrm{Nu}_{1}^{\prime}$ curves do not display discontinuities and negative values. For higher values of $\mathrm{Br}^{*}$, the Nusselt numbers depend very little of $n$, with viscous dissipation overlapping the effect of the large velocity profile variation with $n$. The behavior of the Nusselt numbers for other values of $\Phi$ is similar to the one presented here. 


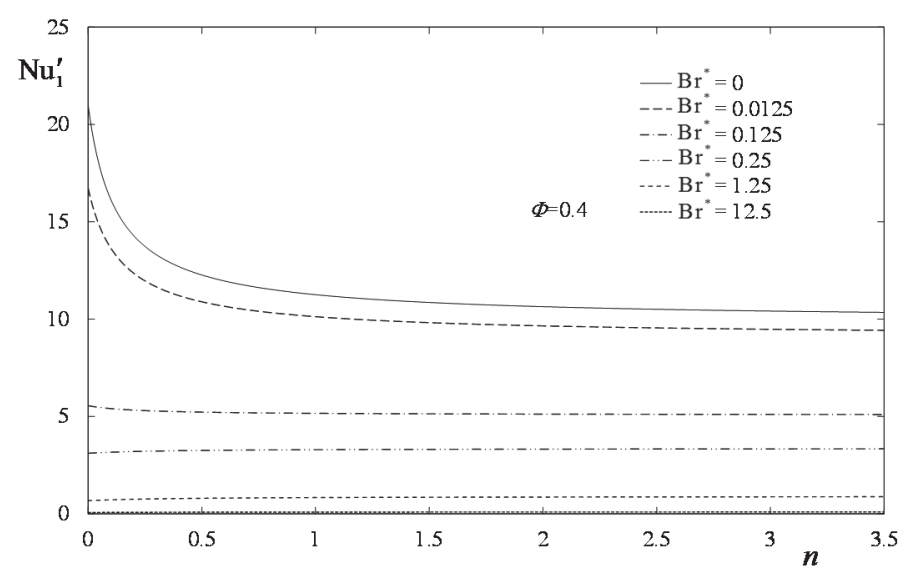

(a) $\mathrm{Nu}_{1}$

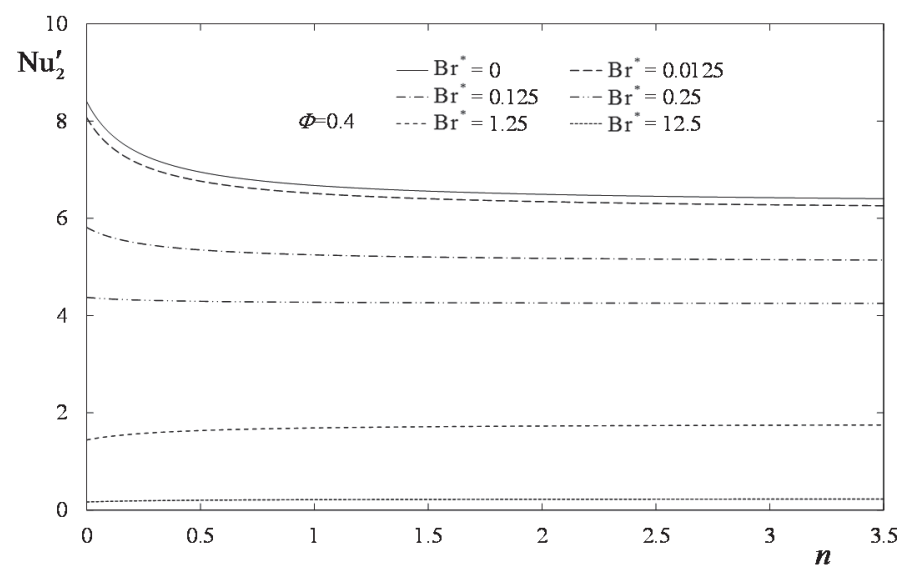

(b) $\mathrm{Nu}_{2}$

Fig. 6 Nusselt numbers versus the power-law index, $n$, for $\Phi=0.4$ and different values of the Brinkman number, $\mathrm{Br}^{*}$. Data obtained using two bulk temperatures, the new approach.

Figure 7 shows the variation of $\mathrm{Nu}_{1}^{\prime}$ and $\mathrm{Nu}_{2}^{\prime}$ with $\Phi$ for different values of the Brinkman number, $\mathrm{Br}^{*}$, and $n=0.5$. For other values of $n$ the plots are very similar to the one presented here. With an increase of $\Phi, \Phi=\dot{q}_{\mathrm{w}, 1} / \dot{q}_{\mathrm{w}, 2}$, the coordinate $y_{\mathrm{I}, d T^{*} / d y^{*}=0}^{*}$ moves away from the wall 1, the temperature difference $T_{\mathrm{w}, 1}^{*}-\bar{T}_{1}^{*}$ increases and $\mathrm{Nu}_{1}^{\prime}$ decreases. On wall 2 the opposite phenomenon occurs and $\mathrm{Nu}_{2}^{\prime}$ increases. In the absence of viscous dissipation, when $\Phi$ tends to zero, i.e., very low values of $\dot{q}_{\mathrm{w}, 1}$, the coordinate $y_{\mathrm{I}, d T^{*} / d y^{*}=0}^{*}$ approaches wall 1 , the value of $T_{\mathrm{w}, 1}^{*}$ tends to the value of the bulk temperature, $\bar{T}_{1}^{*}$, and $\mathrm{Nu}_{1}$ tends to infinity.

For $\Phi<0.2$, when the coordinate $y_{\mathrm{I}, d T^{*} / d y^{*}=0}^{*}$ approaches wall 1 , the temperature profile 1 is also closer to that wall and to the zone where the heat generated by viscous dissipation occurs, cf. Fig. 1. Because of that, the Nusselt number, $\mathrm{Nu}_{1}^{\prime}$, also starts to depend strongly on the Brinkman number, $\mathrm{Br}^{*}$, as shown in Fig. 7a). In the "classical" approach, this dependence goes unnoticed, cf. Fig. 4a).

For higher values of the Brinkman number, the value of $\mathrm{Nu}_{1}^{\prime}$ decreases monotonically with a reduction of $\Phi$, i.e., an increase of $y_{\mathrm{I}, d T^{*} / d y^{*}=0}^{*}$. For small values of the Brinkman number, $\mathrm{Br}^{*}<0.125$ for $n=0$ or $\mathrm{Br}^{*}<0.131$ for $n=2$ for example, the viscous dissipation effect only surpasses the effect of a decrease in $\Phi$, and the corresponding approximation of $T_{\mathrm{w}, 1}^{*}$ to $\bar{T}_{1}^{*}$, for very low values of $\Phi, \Phi<0.05$. This leads to a local maximum of the Nusselt number, for a given value of $\Phi$, or $y_{\mathrm{I}, d T^{*} / d y^{*}=0}^{*}$, as it can be seen in Fig. 7a) for $\mathrm{Br}^{*}=0.0125$.

Figure 8 shows the variation of $\mathrm{Nu}_{1}^{\prime}$ and $\mathrm{Nu}_{2}^{\prime}$ with $\mathrm{Br}^{*}$ for different values of $\Phi$ and $n=0.5$. For other values of $n$ the behaviour is similar. As expected, it is clear that the heat transfer coefficient decreases when the Brinkman number increases, since the heat generated by viscous dissipation hinders the heat transfer.

Once again, it can be seen in Fig. 8a), by contrast with Fig. 8b), that for small values of $\Phi$, i.e., when $y_{\mathrm{I}, d T^{*} / d y^{*}=0}^{*}$ is close to the wall 1 , the corresponding Nusselt number, $\mathrm{Nu}_{1}^{\prime}$, is very sensitive to variations in the Brinkman number. The higher values of the Nusselt numbers are attained with simultaneously lower values of $\Phi$ and $\mathrm{Br}^{*}$. As expected, for $\Phi=1$ the variations of $\mathrm{Nu}_{1}^{\prime}$ and $\mathrm{Nu}_{2}^{\prime}$ with $\mathrm{Br}^{*}$ are the same.

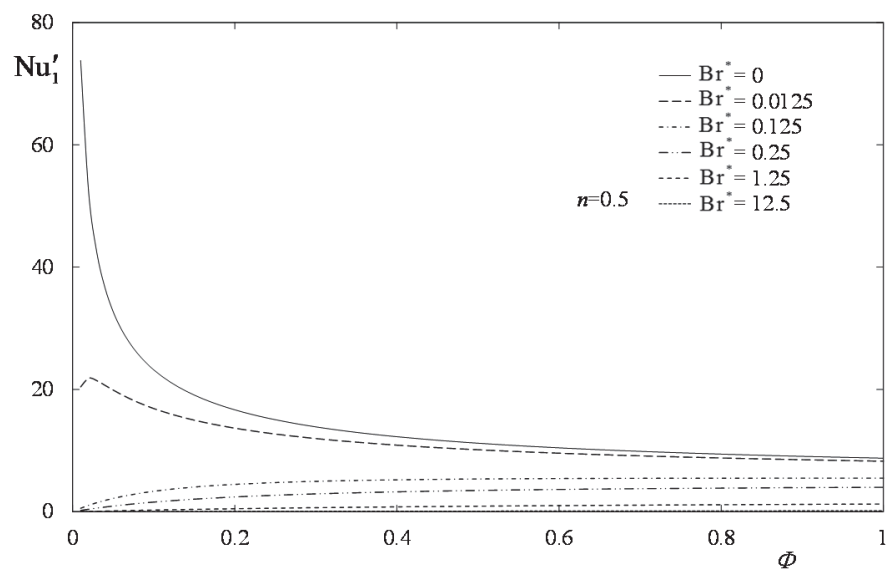

(a) $\mathrm{Nu}_{1}$

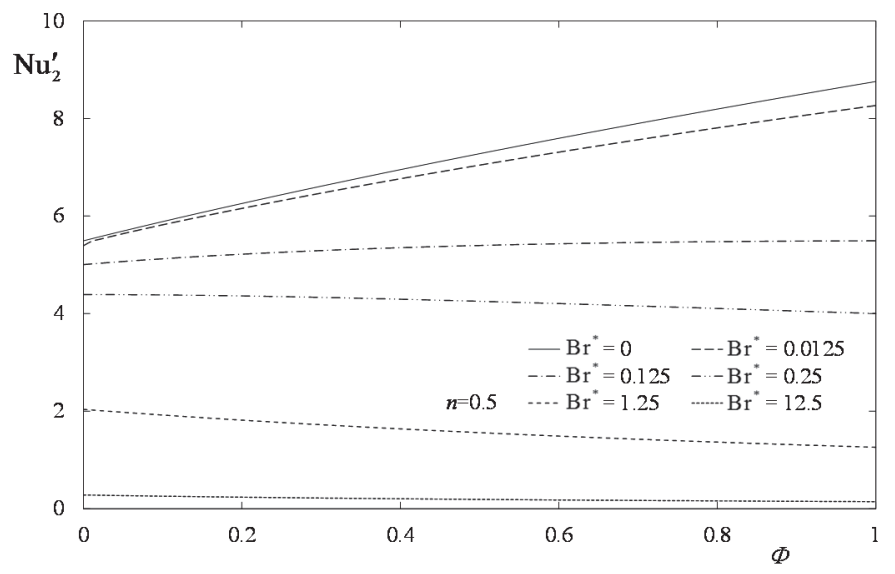

(b) $\mathrm{Nu}_{2}$

Fig. 7 Nusselt numbers versus the wall heat flux ratio, $\Phi$, for $n=0.5$ and different values of the Brinkman number, $\mathrm{Br}^{*}$. Data obtained using two bulk temperatures, the new approach.

Both approaches produce $\mathrm{Nu}$ values at a given wall that are more similar to each other the further away from that wall the coordinate $y_{d T^{*} / d y^{*}=0}^{*}$ is, as seen in Fig. 9 where the variation of $\mathrm{Nu}_{1}$ and $\mathrm{Nu}_{1}^{\prime}$ with the coordinate of the corresponding location of the temperature profile where $\partial T / \partial y=0, y_{\mathrm{I}, d T^{*} / y^{*}=0}^{*}$, is shown. As expected, when $y_{\mathrm{I}, d T^{*} / y^{*}=0}^{*}=0$ the two approaches have the same result since in this 
case the temperature profiles are symmetrical. The further away from the wall 1 the coordinate $y_{\mathrm{I}, d T^{*} / y^{*}=0}^{*}$ is, the closer to each other are the values of the bulk temperatures given by the new approach, $\bar{T}_{1}^{*}$, and by the "classical" approach, $\bar{T}^{*}$, thus the $\mathrm{Nu}$ values given by both approaches also become closer to each other.

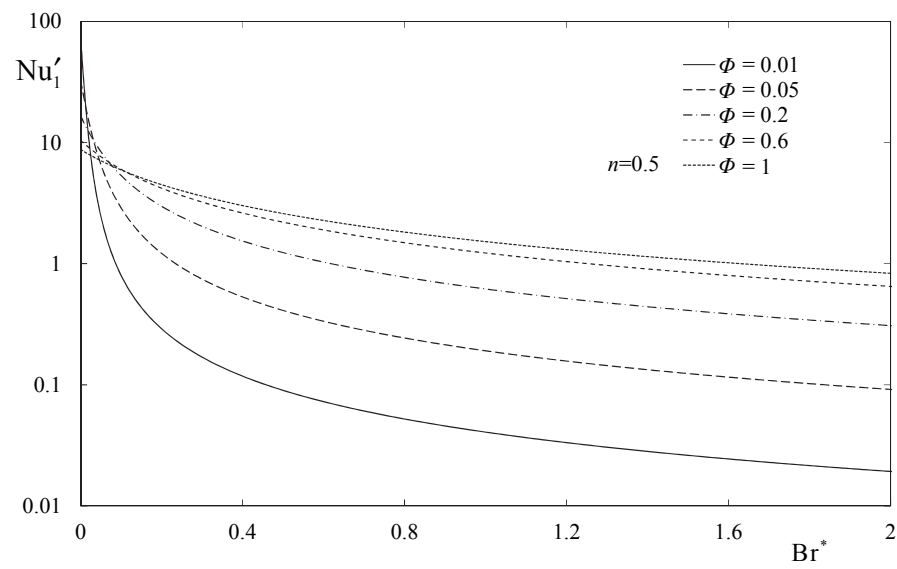

(a) $\mathrm{Nu} 1$

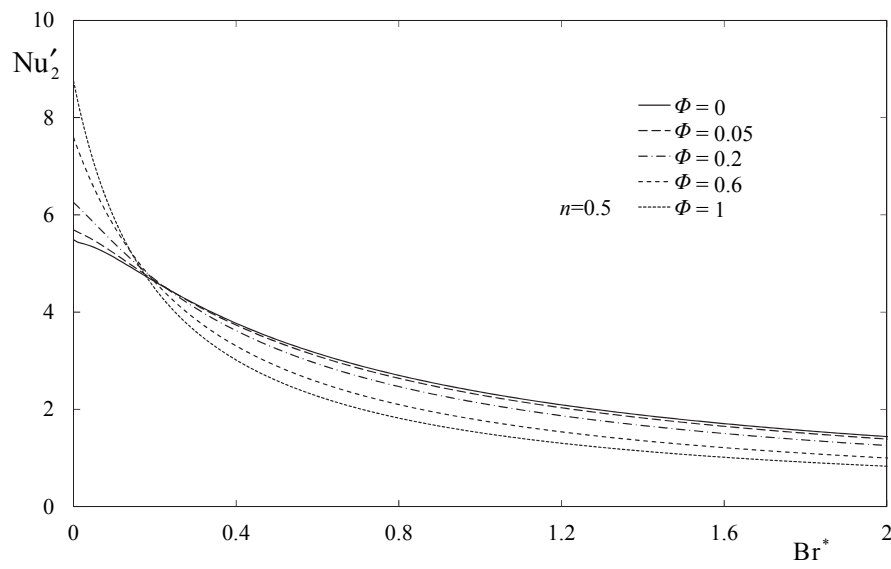

(b) $\mathrm{Nu}_{2}$

Fig. 8 Nusselt numbers versus the Brinkman number, $\mathrm{Br}^{*}$, for $n=0.5$ and different values of the wall heat flux ratio, $\Phi$. Data obtained using two bulk temperatures, the new approach.

In section 3 it was found that for $\mathrm{Br}^{*}=0.25$, both Nusselt numbers were constant, $\mathrm{Nu}_{1}=\mathrm{Nu}_{2}=4$, and simultaneously independent of $\Phi$ and $n$. This singular case is inherent to the "classical" approach and does not occur in this new and more realistic approach.

\section{CONCLUSIONS}

In this work, analytical expressions for the Nusselt number in a laminar flow of a power-law fluid between parallel plates were obtained. These results are valid for a fully developed flow, with constant and different heat fluxes at the walls in the presence of viscous dissipation. In these analytical solutions the "classical" approach was used, i.e., both Nusselt numbers are, as usual, based in the same bulk temperature calculated for the entire duct cross-section. This approach occasionally leads to negative values and discontinuities in the Nusselt number plots, and for the particular case of the Brinkman number, $\mathrm{Br}^{*}$, equal to 0.25 ( $\dot{q}_{\text {visc dissip }} / \overline{\dot{q}}_{\mathrm{w}}=2$ ) a singular result is obtained, i.e., $\mathrm{Nu}_{1}=\mathrm{Nu}_{2}=4$ regardless of the wall heat flux ratios, $\Phi$, and the power-law index, $n$, values.

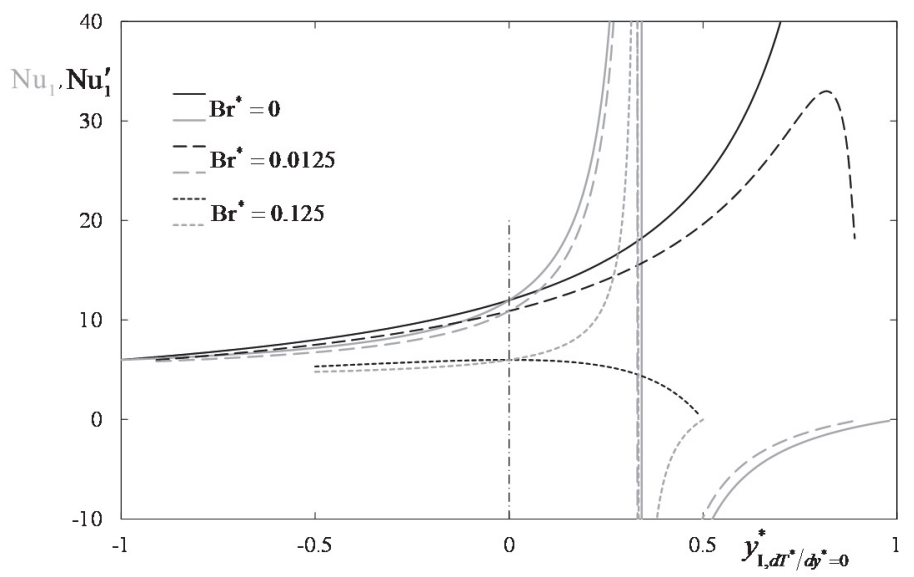

Fig. 9 Nusselt number, $\mathrm{Nu}_{1}$, versus the corresponding coordinate of the location of the temperature profile where $\partial T / \partial y=0, y_{\mathrm{I}, d T^{*} / y^{*}=0}^{*}$, for different values of the Brinkman number, $\mathrm{Br}^{*}$, and $n=0$. Lines: Grey lines - "classical" approach, black lines - new approach, the vertical line marks the duct axis coordinate..

It was found that the temperature profile derivative at the duct axis only depends of $\Phi$, being independent of the values of $n$ or $\mathrm{Br}^{*}$ since the heat transfer across the duct cross-section is symmetrically affected by these two variables. In the particular case in which the heat supplied at the duct walls is equal the heat generated by viscous dissipation, i.e., $\mathrm{Br}^{*}=0.125$, the temperature value, $T^{*}$, at the duct axis is independent of the power law index, $n$

Generally, for low values of $\mathrm{Br}^{*}$, the decrease in the value of $n$ leads the velocity profile to become closer to the plug profile, i.e., greater velocities near the walls, and the Nusselt number increases. For higher values of $\mathrm{Br}^{*}$, the heat generated by viscous dissipation, that approaches the walls when $n$ decreases, surpasses the effect of the increased velocity near the walls and $\mathrm{Nu}$ decreases.

The Nusselt numbers obtained using the traditional approach do not always reflect the anticipated behaviour of the real heat transfer coefficient. In order to obtain $\mathrm{Nu}^{\prime}$ values that are closer to the real ones, and comparable with the literature values for the many other cases where the temperature profile is symmetric, an new approach for the Nusselt numbers determination was also undertaken. The calculation is made using two different bulk temperatures, $\bar{T}_{1}$ and $\bar{T}_{2}$, one for each side of the temperature profile where $\partial T / \partial y=0$, with the transverse coordinate $y=y_{\partial T / \partial y=0}$, avoiding the anomalies discussed above. Both approaches allow the correct calculation of the wall temperature, once known the corresponding Nusselt number and bulk temperature of each approach. The advantage of this new approach is that it also allows the calculation of a more realistic heat transfer coefficient, comparable with the current values in the literature for flows inside ducts having symmetrical temperature profiles, although at the expense of a bulk temperature calculation that is not as straightforward which may hinder its practical implementation.

The Nusselt number plots, obtained using the new approach, do not show discontinuities or negative values. Also from a didactic point of view, it is relevant to show that negative values of Nusselt numbers and discontinuations in the curves that reflect their behavior are not inevitable and that there are ways to avoid this apparently unrealistic behavior.

Another thing that this new approach revealed was the strong variation of the Nusselt numbers with the Brinkman number, at the wall with lower heat flux, for $\Phi$ values smaller than about 0.2 . Finally the 
two different approaches produce $\mathrm{Nu}$ values in a given wall much more similar to each other the further away from that wall the coordinate $y_{d T^{*} / d y^{*}=0}^{*}$ is.

\section{ACKNOWLEDGMENTS}

The help of Prof. Rob J. Poole (Univ. Liverpool) for proofreading the original manuscript and the helpful comments of the anonymous reviewers are gratefully acknowledged.

\section{NOMENCLATURE}

$b \quad$ duct spanwise length (m)

$\mathrm{Br}^{*} \quad$ generalized de Brinkman, $\mathrm{Br}^{*}=\bar{U} \tau_{\mathrm{w}} /\left(8 \overline{\dot{q}}_{\mathrm{w}}\right)$

c specific heat of the fluid $\left(\mathrm{J} / \mathrm{m}^{3} \cdot \mathrm{K}\right)$

$D_{\mathrm{h}} \quad$ duct hydraulic diameter, $D_{\mathrm{h}}=4 H(\mathrm{~m})$

$h \quad$ convective heat transfer coefficient $\left(\mathrm{W} / \mathrm{m}^{2} \cdot \mathrm{K}\right)$

$H \quad$ half distance between parallel plates (m)

$k \quad$ thermal conductivity of the fluid $(\mathrm{W} / \mathrm{m} \cdot \mathrm{K})$

$n$ power law index

$\mathrm{Nu} \quad$ Nusselt number, $\mathrm{Nu}=h D_{\mathrm{h}} / k$

$\mathrm{Nu}^{\prime} \quad$ Nusselt number, new approach

Pe Péclet number, $\mathrm{Pe}=\rho \bar{U} D_{\mathrm{h}} c / k$

$\overline{\dot{q}}_{\mathrm{w}} \quad$ Average wall heat flux, $\overline{\dot{q}}_{\mathrm{w}}=\left(\dot{q}_{\mathrm{w}, 1}+\dot{q}_{\mathrm{w}, 2}\right) / 2\left(\mathrm{~W} / \mathrm{m}^{2}\right)$

$\dot{q}_{\mathrm{w}} \quad$ heat flux at the wall $\left(\mathrm{W} / \mathrm{m}^{2}\right)$

$T^{*} \quad$ nondimensional temperature, $T^{*}=\left(T-T_{0}\right) k /\left(D_{\mathrm{h}} \overline{\dot{q}}_{\mathrm{w}}\right)$

$\bar{T} \quad$ bulk temperature $\mathrm{K}$

$T \quad$ local temperature $\mathrm{K}$

$T_{0} \quad$ reference bulk temperature $\mathrm{K}$

$\bar{U} \quad$ bulk velocity $(\mathrm{m} / \mathrm{s})$

$u^{*} \quad$ nondimensional local velocity, $u^{*}=u / \bar{U}$

$x \quad$ longitudinal coordinate $(\mathrm{m})$

$x^{*} \quad$ nondimensional longitudinal coordinate, $x^{*}=x / D_{\mathrm{h}}$

$y \quad$ coordinate normal to the parallel plates $(\mathrm{m})$

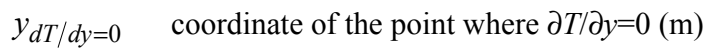

$y^{*} \quad$ nondimensional coordinate normal to the wall, $y^{*}=y / H$

\section{Greek Symbols}

$\alpha \quad$ compacting constant, $\alpha=2 \operatorname{Br}^{*}(2 n+1) / n$

$\beta \quad$ compacting constant, $\beta=\left(1+8 \mathrm{Br}^{*}\right)(2 n+1) /(4 n+4)$

$\varepsilon \quad$ compacting constant, $\mathcal{\varepsilon}=(n+1) / n$

$\varphi \quad$ compacting constant, $\varphi=1 /[2(\Phi+1)]$

$\sigma \quad$ compacting constant, $\sigma=(\alpha-\beta \varepsilon) /(\varepsilon+1)$

$\eta \quad$ compacting constant, $\eta=(\alpha+\beta) /(\varepsilon+1)$

$\rho \quad$ density of the fluid $\left(\mathrm{kg} / \mathrm{m}^{3}\right)$

$\boldsymbol{\Phi} \quad$ wall heat flux ratio, $\boldsymbol{\Phi}=\dot{q}_{\mathrm{w}, 1} / \dot{q}_{\mathrm{w}, 2}$ $\tau^{*} \quad$ nondimensional shear stress, $\tau^{*}=\tau_{y x} / \tau_{\mathrm{w}}=y^{*}$

$\tau_{\mathrm{w}} \quad$ wall shear stress, absolute value $(\mathrm{Pa})$

$\tau_{y x} \quad$ local shear stress, absolute value $(\mathrm{Pa})$

Subscripts

1 relative to wall 1 , cf. Fig. 1

2 relative to wall 2 , cf. Fig. 1

I relative to region I, cf. Fig. 1

II relative to region II, cf. Fig. 1

w wall

\section{REFERENCES}

Baptista A., Alves M.A., Coelho P.M., 2013, "Heat Transfer in Fully Developed Laminar Flow of Power Law Fluids," J. Heat Transf, 136(4), 041702.

http://dx.doi.org/10.1115/1.4025662.

Çengel, Y. A., and Turner, R. H., 2005, Fundamentals of ThermalFluid Sciences, 2nd ed., McGraw-Hill, New York.

Coelho, P.M. and Faria, J. C., 2011, "On the Generalized Brinkman Number Definition and Its Importance for Bingham Fluids," ASME Trans J Heat Transf, 133(5), 054505-1. http://dx.doi.org/10.1115/1.4003169.

Coelho, P.M., and Pinho, F. T., 2009, "A Generalized Brinkman Number for Non Newtonian Duct Flows," J. Non-Newtonian Fluid Mech., 156, 202-206.

http://dx.doi.org/10.1016/j.jnnfm.2008.07.001.

Coelho, P. M. and Poole, R. J., 2017, "Heat Transfer of Bingham Fluids in an Annular Duct with Viscous Dissipation," Heat Transfer Engineering, paper accepted for publication.

Etemad, S.Gh., Majumdar, A.S. and Huang, B., 1994, "Viscous Dissipation Effects In Entrance Region Heat Transfer For A Power Law Fluid Flowing Between Parallel Plates," Int. J. Heat Fluid Flow, 15, 122-131.

https://doi.org/10.1016/0142-727X(94)90066-3.

Hartnett, J. P. and Kostic, M., 1989, "Heat Transfer to Newtonian and Non-Newtonian Fluids in Rectangular Ducts," Advances in Heat Transfer, 19, 247-356.

https://doi.org/10.1016/S0065-2717(08)70214-4.

Lawal, A.; Mujumdar, A. S., 1987, Laminar non-Newtonian Flow and Heat Transfer in Ducts, Advances in transport processes (A. S. Mujumdar and R. A. Mashelkar, ed.). Wiley Eastern Ltd., India, 5, 351442.

Shah, R.K. and London, A.L., 1978, Laminar Flow Forced Convection in Ducts, Academic Press, New York.

Skelland, A. H. P., 1967, Non-Newtonian Flow and Heat Transfer, Wiley, New York.

Tso, C.P., Sheela-Francisca, J. and Yew-Mun Hung, 2010, "Viscous Dissipation Effects of Power-law Fluid Flow within Parallel Plates with Constant Heat Fluxes," J. Non-Newton. Fluid Mech., 165, 625-630. http://dx.doi.org/10.1016/j.jnnfm.2010.02.023. 